\section{The unusual suspects}

The pathogenesis of type 1 diabetes (T1D) is well established, but the initial triggers of the disease remain unclear. In Nature Medicine, Lehuen and colleagues attribute critical roles to a trio of cells of the innate immune system in the initiation of T1D. The authors observe infiltration of B-1a cells, neutrophils and plasmacytoid dendritic cells into the pancreatic islets of T1D-prone mice of the nonobese diabetic (NOD) strain. This innate-cell infiltrate is notably absent from mouse strains not prone to autoimmune disease. This is because dying cells in the islets are normally cleared by macrophages, but in NOD mice, this fails to occur, which leads to the appearance of antibodies to DNA from the B-1a cells. Those antibodies in turn activate the neutrophils to release extracellular traps and antimicrobial peptides. Those released factors not only cause further damage but also trigger plasmacytoid dendritic cells through TLR9 to release type I interferon, which has known roles in many autoimmune diseases.

Nat. Med. 19, 65-73 (2013)

\section{Living with commensals}

The intestinal microbiota is tolerated by the host, and potentially harmful inflammatory responses to commensals could be actively suppressed. In Nature, Littman and colleagues show that, at steady state, the microbiota inhibits the transport of commensal and pathogenic bacteria from the intestinal lumen to the mesenteric lymph nodes (MLNs). After antibioticinduced dysbiosis, noninvasive Salmonella enterica or nonpathogenic Escherichia coli, which under normal conditions do not reach the MLNs, are trafficked to the MLNs by CX3CR 1 hi phagocytes in a chemokine receptor CCR7-dependent manner. Mice deficient in the adaptor Myd88 similarly allow trafficking of bacteria to the MLNs and induction of specific $\mathrm{T}$ cell responses and production of $\operatorname{Ig} \mathrm{A}$, which indicates that sensing the microbiota via TLRs inhibits a pathway that allows access of lamina propria cell populations and phagocytosed bacteria to the MLNs.

Nature (13 January 2013) http://dx.doi.org/10.1038/nature11809

\section{Clipping B cells}

$B$ cells arise in the bone marrow and emerge as immature cells that further develop in the spleen and lymph nodes. In the Journal of Experimental Medicine, Schröder, Enders and Cooke identify a requirement for intramembrane cleavage of the invariant chain li (CD74) for B cell maturation. The endopeptidase SPPL2A, a membrane-tethered protease, is required for this intramembrane cleavage of CD74. Mutant mice that lack active SPPL2A have fewer mature B cells and lower titers of serum IgG and fail to mount antigen-specific antibodies after immunization. B cell development is arrested at the transitional $\mathrm{T} 1$ stage, as B cells fail to upregulate BCR and BAFF receptors. Instead, SPPL2A-deficient B cells accumulate $\mathrm{N}$-terminal fragments of CD74 in endosomal vesicles, which impedes normal vesicular trafficking of endosomal cargo and thereby compromises the ability of the cells to receive prosurvival signals. Although these three reports identify a role for SPPL2A in $B$ cell maturation, whether antibody-secreting plasma cells likewise require SPPL2A function remains unknown.

J. Exp. Med. 210, 23-30, 31-40 \& 41-58 (2013)

\section{Latent enhancement}

The enhancer repertoire that defines the functional phenotype of a terminally differentiated cell is maintained by the coordinate action of transcription factors and characteristic histone marks. In Cell, Natoli and colleagues identify so-called 'latent enhancers' with unique epigenetic characteristics. In macrophages, they find that latent enhancers are not bound by transcription factors and lack any known histone marks under resting conditions; however, those features are acquired after treatment with a variety of stimuli, such as exposure to lipopolysaccharide. Removal of the stimuli followed by resting of macrophages 'scrubs' transcription factors and most epigenetic marks from latent enhancers, except for histone $\mathrm{H} 3$ monomethylated at Lys4, indicative of an enhancer in a poised state. Subsequent restimulation of those macrophages leads to more rapid histone acetylation and gene induction. Therefore, latent enhancers can 'scar' the epigenetic landscape of a cell and provide a form of 'stimulus history' that could explain aspects of macrophage plasticity and innate immune cell 'memory'.

Cell 152, 157-171 (2012)

\section{Gender, microbes \& autoimmunity}

Gender biases are prevalent in many autoimmune syndromes. In Science, Danska and colleagues explore how gender influences disease manifestation in NOD mice. Female NOD mice have a higher incidence of disease than do male NOD mice, but this bias disappears when the mice are raised in germ-free facilities. Metabolomics also identifies differences between male and female mice in their circulating glycerophospholipids and sphingolipid long-chain fatty acids. Gender-specific differences in microbial populations arise during puberty. The transfer of microbiota from adult male mice to young female mouse pups confers protection against diabetogenesis in this NOD model. Male-to-female microbiome transfer leads to an increase in circulating testosterone in the recipients and fewer differences in circulating metabolytes. These findings suggest that sex hormones influence microbial populations and reciprocatively influence systemic metabolism that affects the development of autoimmune diabetes.

$L A D$

Science (17 January 2013) http://dx.doi.org/10.1126/

science. 1233521

\section{Signal integration}

Both stable and unstable interactions between $\mathrm{T}$ cells and antigenpresenting cells (APCs) have been observed in vivo, but the functional importance of these two types of contacts for $\mathrm{T}$ cells activation remains unclear. In Immunity, Marangoni et al. show that transient versus sustained T cell-APC interactions can be 'translated' into differences in gene activation in a manner dependent on the protracted nuclear retention of NFAT transcription factors. Contact of $\mathrm{CD}^{+} \mathrm{T}$ cells with APCs induces fast accumulation of NFAT in the nucleus (half-life, $\sim 1 \mathrm{~min}$ ), whereas export of NFAT from the nucleus is slow (half-life, $\sim 20 \mathrm{~min}$ ). During that 'signal memory' phase, NFAT remains transcriptionally active for the tolerance-associated gene Egr2 but not for the effector cytokine-encoding gene Ifng, which requires active signaling via the TCR for expression. As regulatory $\mathrm{T}$ cells can impair stable contacts between effector $\mathrm{T}$ cells and APCs, unstable interactions and retention of NFAT in the nucleus may represent a mechanism for inducing tolerance. Immunity (10 January 2013) http://dx.doi.org/10.1016/j. immuni.2012.09.012 